\title{
Aplicación de metamateriales para estimar índices de refracción
}

\author{
다 Javier Ricardo Castro-Ladino ${ }^{1}$, 고 Alba G. Ávila-Bernal ${ }^{1,2, *}$ \\ ${ }^{1}$ Escuela de Ingeniería, Universidad de los Llanos, Villavicencio, Colombia, \\ ${ }^{2}$ Departamento de Ingeniería Eléctrica y Electrónica, Universidad de los Andes, Bogotá D.C., Colombia
}

\begin{abstract}
Resumen
Se presenta el desarrollo de un metamaterial para estimar el índice de refracción en líquidos. El metamaterial se desarrolló insertando celdas cilíndricas en el plano de tierra de una línea microcinta, cintas estas que forman una estructura de brecha de banda electromagnética (electromagnetic bandgap, EBG). Dentro de las celdas se depositaron líquidos con índices de refracción estimados en el laboratorio. Se evaluaron los parámetros de dispersión $S_{21}$ y $S_{11}$ mediante simulaciones electromagnéticas con modelos desarrollados, y de forma experimental, con prototipos fabricados. Se analizó la variación de la frecuencia central, el ancho de banda y el nivel de pérdidas por inserción de la brecha de frecuencias generada como respuesta a la variación del índice de refracción del material depositado dentro de las celdas. Los resultados obtenidos muestran que es posible estimar el índice de refracción de materiales monitoreando la frecuencia de resonancia o el nivel de pérdidas por inserción de la brecha generada. El metamaterial desarrollado es fácil de construir y su desempeño es adecuado en el rango de las microondas. C) 2019. Acad. Colomb. Cienc. Ex. Fis. Nat.
\end{abstract}

Palabras clave: Metamateriales; EBG; Índice de refracción.

Application of metamaterials to estimate refractive indexes

\begin{abstract}
We developed a metamaterial to estimate the refractive index in liquids by inserting cylindrical cells in the ground plane of a microstrip line, lines that form an electromagnetic bandgap (EBG) structure. We deposited inside the cells liquids with refractive indices estimated in the laboratory. The dispersion parameters $S_{21}$ and $S_{11}$ were evaluated by means of electromagnetic simulations with developed models and experimentally with manufactured prototypes. We analyzed the variation of center frequency, the bandwidth, and the level of losses by the variation of the refractive index of the material deposited inside the cells. Our results showed that it is possible to estimate the refractive index of materials by monitoring the resonance frequency or the level of insertion losses of the generated gap. The metamaterial developed is easy to build and its performance is adequate in the range of microwaves. (C) 2019. Acad. Colomb. Cienc. Ex. Fis. Nat.
\end{abstract}

Key words: Metamaterials; EBG; Refractive index sensor.

\section{Introducción}

La determinación de las características de un material, tales como la permitividad, la permeabilidad y la constante dieléctrica, es importante en aplicaciones utilizadas, por ejemplo, en los procesos médicos e industriales para sólidos y líquidos (Sadat, 2017), y en la industria de alimentos y de ingeniería civil, entre otras (Domínguez, 2015). El índice de refracción es una característica inherente de una sustancia, estrechamente relacionada con sus propiedades físicas y químicas (Yüksel, 2016). Varias técnicas se han desarrollado para medir estas características como indicadores de los cambios químicos y físicos de los materiales.

Debido a sus características, entre las que se destacan la precisión, el bajo consumo de energía y el bajo costo, los sensores de microondas se han utilizado para medir las propiedades de los materiales (Majidifar, 2016). Se han analizado líquidos, gases y materiales sólidos mediante sensores de microondas, con lo que se ha demostrado su amplia aceptabilidad (Domínguez, 2015). Los metamateriales (MTM) son apropiados para el desarrollo de sensores de microondas. Son materiales artificiales obtenidos mediante la combinación de diferentes tipos de estructuras o materiales, capaces de controlar simultáneamente la permitividad y la permeabilidad, que han demostrado tener propiedades deseables para una amplia gama de aplicaciones en ingeniería que no ofrecen los materiales en su estado natural (Cimelli, 2011). Estas estructuras son atractivas para el desarrollo de nuevos componentes y subsistemas en diferentes áreas,

\footnotetext{
*Correspondencia:

Alba G. Ávila-Bernal; a-avila@uniandes.edu.co

Recibido: 6 de marzo de 2019

Aceptado: 15 de mayo de 2019

Editor: Diógenes Campo
} 
entre las que se destacan las comunicaciones y los sensores (Lapine, 2009; Bakir, 2017; Tümkaya, 2018), las aplicaciones biomédicas, el monitoreo de procesos de materiales compuestos y los biosensores (Shengyon, 2018).

El principio básico de funcionamiento de la detección con metamateriales se basa en el cambio de los coeficientes de transmisión-reflexión ( $S$ - parámetros de dispersión) en términos de frecuencia o factor de calidad producto de un cambio en los parámetros detectados, por ejemplo, el cambio dieléctrico del medio por el cual se transmite una señal, la variación en $\mu / \varepsilon$ o el índice de refracción del material (Chen, 2012; RoyChoudhury, et al., 2016).

Se han desarrollado varios sensores utilizando las propiedades de los metamateriales, especialmente biosensores y sensores de microondas en diversas aplicaciones para determinar la presión, la temperatura, la humedad y la permitividad (Bakir, 2017; Sadat, 2017; Domínguez, 2015; Chen, 2012), así como el índice de refracción (Fei, et al., 2015) y la concentración de nanopartículas (Liu, et al., 2017), entre otras. Los metamateriales desarrollados en forma de una línea de microcinta insertando estructuras EBG formadas por celdas cilíndricas (3D) en el plano de tierra, son fáciles de construir, requieren pocos recursos técnicos $\mathrm{y}$ económicos, y tienen un rendimiento adecuado en el rango de las microondas.

Como consecuencia de la inserción de las estructuras EBG, el metamaterial desarrollado atenúa las señales de microondas transmitidas en un cierto rango de frecuencia (brecha) debido a la variación del índice de refracción sobre la misma capa de sustrato dieléctrico. Cuando una onda incide sobre un material, el cambio en su respuesta electromagnética se rige por dos parámetros fundamentales: la permitividad $(\varepsilon)$ y la permeabilidad $(\mu)$ (RoyChoudhury, et al., 2016). Los valores de $\varepsilon$ y $\mu$ están relacionados con el índice de refracción mediante la siguiente ecuación:

$$
n=\sqrt{\varepsilon_{r} \mu_{r}}
$$

La frecuencia central $\left(f_{c}\right)$ de la brecha generada se puede estimar a partir de la condición de difracción de Bragg hasta el primer orden, que establece que para que exista reflexión se debe cumplir con la condición de que la distancia interplanar o la separación entre los defectos sea igual a la mitad de la longitud de onda $(d=\lambda / 2)$. Cuando esta relación se cumple, las frecuencias se atenúan impidiendo la propagación de la señal en este rango. La $f_{c}$ de la brecha generada y la constante de red $(d)$ de la estructura EBG, se pueden calcular a partir de la constante de propagación de la onda en el sustrato dieléctrico, que se define como:

$$
\beta=\frac{2 \pi f_{c} \sqrt{\varepsilon_{r}}}{c}, \quad \beta=2 \pi \lambda, \quad f_{c}=\frac{c}{2 d \sqrt{\varepsilon_{r}}}
$$

En consecuencia, es posible estimar el índice de refracción de materiales depositados dentro de las celdas de la estructura EBG monitoreando la variación de la $f_{c}$ de la brecha generada.

En este trabajo se presenta el desarrollo experimental de un metamaterial para estimar el índice de refracción en líquidos depositados dentro de las celdas de una estructura EBG ubicada en el plano de tierra de una línea microcinta construida sobre un sustrato dieléctrico. El índice de refracción se estimó monitoreando los cambios en la $f_{c}$ o el nivel de pérdidas por inserción (IL) de la brecha generada.

\section{Materiales y métodos}

Se desarrolló un metamaterial al introducir una estructura EBG conformada por celdas cilíndricas $3 \mathrm{D}$ con $8 \mathrm{~mm}$ de radio $(r=8 \mathrm{~mm})$ y $1 \mathrm{~mm}$ de profundidad $\left(h_{C}=1 \mathrm{~mm}\right)$, ubicada en el plano de tierra de una línea microcinta sobre sustratos dieléctricos FR4 y RT/duroid® 6010 (Rogers Corporation), respectivamente (Figura 1), utilizando técnicas de fabricación de circuitos impresos y de fresado con equipo de control numérico (CNC).

El modelo se simuló en el programa HFSS (Ansoft), ubicando dentro de las celdas cilindros de $200 \mu \mathrm{m}$ de altura $\left(h_{C}=200 \mu \mathrm{m}\right)$ para simular el depósito de cinco líquidos con diferentes índices de refracción $(n)$. El índice de refracción (IR) de cada líquido se estimó con un refractómetro Modelo 10450 de American Optical, con rango de medida de 1,32 a 1,7 (Tabla 1). Se tomó como referencia el aceite de inmersión

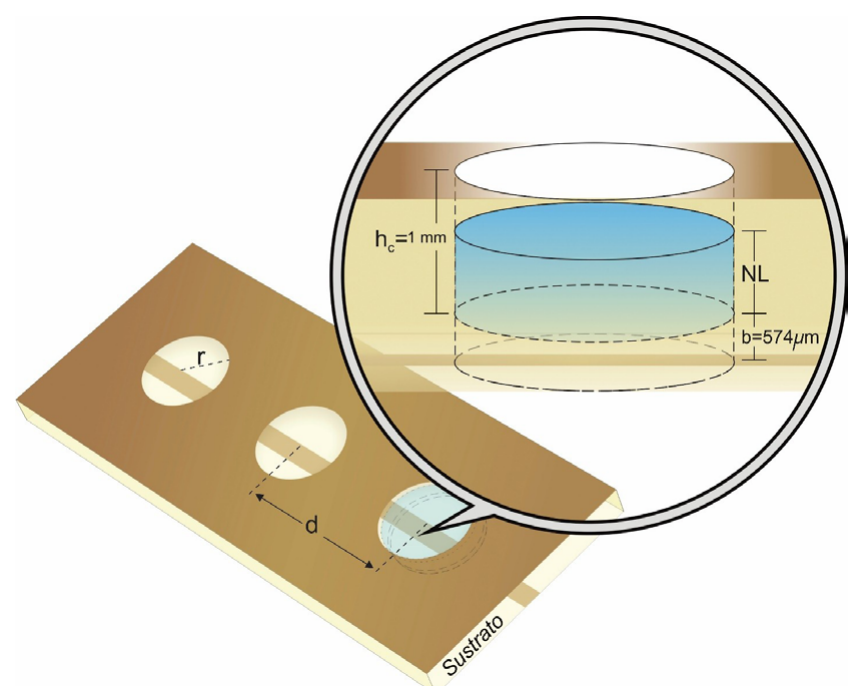

Figura 1. Diagrama esquemático de la línea microcinta con estructura EBG en celdas cilíndricas ubicadas en el plano de tierra

Tabla 1. Índices de refracción estimados

\begin{tabular}{lcc}
\hline Líquido & $\begin{array}{c}\text { Temperatura } \\
\left({ }^{\circ} \mathbf{C}\right)\end{array}$ & $\begin{array}{c}\text { Índice de } \\
\text { refracción } \\
\text { estimado }\end{array}$ \\
\hline Agua estilada & 25,5 & 1,333 \\
\hline Acetona & 24,8 & 1,357 \\
\hline Butanol & 25,0 & 1,398 \\
\hline Glicerina & 25,2 & 1,471 \\
\hline Aceite de inmersión de tipo $\mathrm{F}$ & 24,8 & 1,515 \\
\hline
\end{tabular}


tipo F para microscopía óptica (Olympus - Immersion Oil Type F), con un índice de refracción $n_{e}=1.518$, a $23{ }^{\circ} \mathrm{C}$, certificado por el fabricante. El índice de refracción de este aceite medido bajo las condiciones de laboratorio descritas fue de $n=5.515$ a $24,8{ }^{\circ} \mathrm{C}$.

Para las simulaciones electromagnéticas se utilizaron los índices de refracción estimados en el laboratorio; el modelo del metamaterial se simuló en un rango de $1 \mathrm{GHz}$ a $5 \mathrm{GHz}$, colocando estructuras EBG conformadas por tres y cinco celdas cilíndricas, con las dimensiones mencionadas y una constante de red de $29 \mathrm{~mm}(d=29 \mathrm{~mm})$ sobre los dos sustratos seleccionados. El resultado de las simulaciones evidenció que el material depositado dentro de las celdas generó una variación en la $f_{c}$, el ancho de banda $(B W)$ y el IL $\left(S_{21}\right.$ - parámetro de dispersión) de la brecha generada (Figura 2).

En la Figura 2 se observa una variación en la $f_{c}\left(\Delta f_{c}\right)$ y en el $I L\left(\Delta S_{21}\right)$ de la brecha generada para cada uno de los cinco líquidos simulados utilizando el sustrato FR4. La respuesta fue similar para los modelos desarrollados y simulados sobre el sustrato RT/duroid ${ }^{\circledR} 6010$, pero con una frecuencia central cercana a 2,6 GHz. Se presentó un corrimiento hacia la izquierda (disminución) de la $f_{c}$ y una disminución en el $I L$ $\left(S_{21}\right)$ como respuesta al incremento del índice de refracción.

La respuesta presentó características similares para los cinco líquidos en los 20 modelos simulados. Al incrementar el número de celdas, la magnitud de la frecuencia central disminuyó en 0,1 $\mathrm{GHz}$ aproximadamente; es decir, el modelo del metamaterial con cinco celdas presentó una $f_{c}$ de la brecha generada menor que la del modelo con tres celdas, pero aumentó el $B W$ en un $12 \%$, y el $I L$ en $20 d B$ aproximadamente. Se fabricó el prototipo utilizando estructuras EBG de tres celdas porque en las respuestas obtenidas en la simulación del modelo con cinco celdas los parámetros de dispersión $S_{11}$ y $S_{21}$ no se interceptaron en las frecuencias superiores a $4 \mathrm{GHz}$.

Los prototipos se fabricaron mediante un proceso de fresado para generar la estructura EBG conformada por tres cilindros de $8 \mathrm{~mm}$ de radio y $1 \mathrm{~mm}$ de profundidad sobre una tarjeta de circuito impreso (PCB) del tipo FR4, con una constante de permitividad de $\varepsilon_{r}=4.4$. El grosor del sustrato fue de $1,57 \mathrm{~mm}$ y el espesor de las capas de cobre, de $17 \mu \mathrm{m}$ (Figura 1S, https://www.raccefyn.co/index.php/raccefyn/ article/downloadSuppFile/850/3839). Utilizando técnicas de litografía, se generó en la cara superior del sustrato una línea microcinta con ancho de $3 \mathrm{~mm}$ e impedancia de 50 $\Omega$. Se eligió el sustrato FR4 por ser más grueso que el RT/ duroid ${ }^{\circledR} 6010$, lo que facilita el proceso de fresado.

Inicialmente se hicieron mediciones con los cinco líquidos seleccionados llenando completamente cada una de las celdas, es decir, a un nivel de $1 \mathrm{~mm}$, lo que corresponde a un volumen de $200 \mu L$ (Castro, 2012). La respuesta de los parámetros de dispersión observada en la pantalla del analizador vectorial de redes (VNA), no correspondía con las curvas esperadas, además, presentaban ondulaciones y

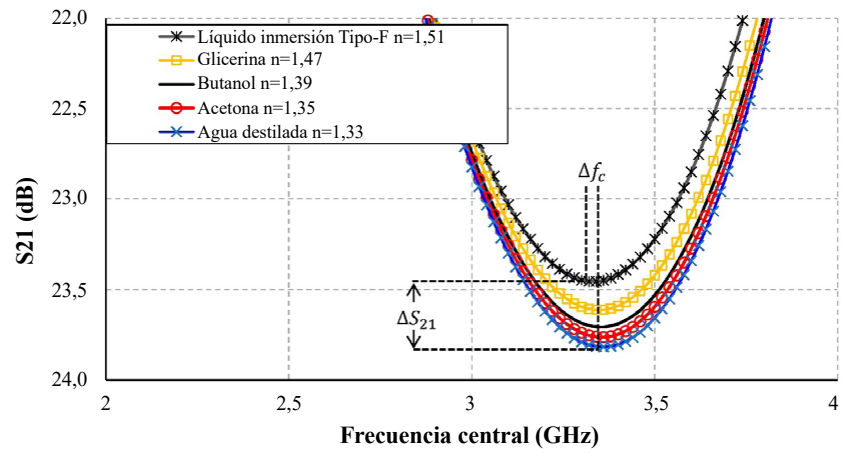

Figura 2. Parámetro de dispersión $S_{21}$ Vs. frecuencia para cada líquido simulado

no se observó una adecuada correspondencia entre la magnitud de los parámetros $S_{11}$ y $S_{21}$. En consecuencia, se realizaron varias mediciones variando el nivel de líquido dentro de las celdas y se encontró que el más adecuado era de $40 \mu \mathrm{L}$. Utilizando este nivel de llenado de las celdas, se procedió a hacer simulaciones electromagnéticas para todos los modelos fabricados y, posteriormente, las mediciones en el laboratorio.

La $f_{c}$ y el $I L$ de la brecha generada se tomaron en el punto correspondiente a la máxima atenuación del parámetro $S_{21}$. Desde ese punto se desplazó el cursor a la misma frecuencia sobre la curva que representaba el parámetro $S_{11}$ y desde este punto se tomaron otros dos, uno a la izquierda y otro a la derecha, que correspondían a una atenuación de $-3 d B$; la diferencia entre estos dos puntos representa el $B W$.

Para cada prueba se midieron los parámetros de dispersión $S_{11}$ y $S_{21}$ antes de adicionar el correspondiente líquido (celdas llenas de aire). Luego se depositaban $40 \mu L$ del líquido dentro de cada una de las celdas, se medían los dos parámetros de dispersión, y se dejaba el montaje durante quince (15) minutos para evaluar la estabilidad de la respuesta. Por último, se retiraba el líquido de las celdas y estas se limpiaban utilizando isopropanol y luego se dejaban secar durante 15 minutos antes de proceder a hacer las mediciones con otro líquido. Para cada prueba se hicieron cinco mediciones en días y horas diferentes monitoreando la temperatura en un punto cercano al prototipo bajo prueba. La temperatura osciló entre 20,5 y $23{ }^{\circ} \mathrm{C}$, con una media de $21,32{ }^{\circ} \mathrm{C}$. En cada medición se tomaron 201 puntos dentro del rango de $1 \mathrm{GHz}$ a $5 \mathrm{GHz}$. Se calculó la media aritmética de los resultados de las cinco mediciones de cada montaje.

El analizador VNA utilizado posee un rango de medida de hasta $6 \mathrm{GHz}$. Cada tarjeta prototipo se ubicó en una mesa de pruebas (Figura 2S, https://www.raccefyn.co/index. $\mathrm{php} /$ raccefyn/article/downloadSuppFile/850/3840) con la estructura PBG en la parte superior para depositar líquidos dentro de cada una de las celdas. Para la manipulación de los líquidos se utilizó una micropipeta digital de volumen variable (Science MED 0200500) de $10 \mu L$ a $100 \mu L$, con incrementos de $1 \mu L$ y una precisión de $0,3 \%$. 
Tabla 2. Magnitud de los parámetros de la brecha generada, y simulación y medición del metamaterial al depositar líquidos dentro de las tres celdas de la estructura EBG

\begin{tabular}{lccccccc}
\hline Líquido & $\begin{array}{c}\text { Índice de } \\
\text { refracción }\end{array}$ & \multicolumn{2}{c}{ Frecuencia central (GHz) } & \multicolumn{2}{c}{ Pérdidas por Inserción - IL (dB) } & \multicolumn{2}{c}{ Ancho de banda } \\
\cline { 5 - 8 } & & Simulado & Medido & Simulado & Medido & Simulado & Medido \\
\hline Agua destilada & 1,333 & 3,36 & 3,28 & 23,82 & 22,95 & 2,58 & 2,50 \\
\hline Acetona & 1,357 & 3,34 & 3,26 & 23,76 & 22,89 & 2,58 & 2,48 \\
Butanol & 1,398 & 3,32 & 3,22 & 23,70 & 22,76 & 2,58 & 2,46 \\
Glicerina & 1,471 & 3,32 & 3,20 & 23,61 & 22,55 & 2,56 & 2,44 \\
Aceite de inmersión de tipo F & 1,515 & 3,30 & 3,20 & 23,45 & 22,37 & 2,54 & 2,42 \\
\hline
\end{tabular}

\section{Resultados}

En la Tabla 2 y la Figura 3 se presentan los resultados de la variación de la magnitud de la $f_{c}$, el $B W$ y el $I L\left(S_{21}\right)$ de la brecha generada al introducir los líquidos seleccionados en las tres celdas de la estructura EBG.

Con los datos obtenidos en las mediciones se elaboraron gráficas de los parámetros de dispersión $S_{11}$ y $S_{21}$ para cada líquido y se compararon los resultados obtenidos en las simulaciones con los obtenidos experimentalmente en el laboratorio. Se ajustó el parámetro $S_{21}$ utilizando herramientas de MatLab (MathWorks ${ }^{\circledR}$ ) debido a las ondulaciones que presentaban las curvas correspondientes a los datos experimentales, causadas, probablemente, por ruido e impurezas presentes en el procedimiento experimental. Se asumió como criterio el seleccionar el polinomio cuya curva presentara la mínima desviación y se calculó el punto máximo de la función. En la Figura 4 se presenta, a manera de ejemplo, el ajuste realizado a las curvas correspondientes al agua destilada y al aceite de inmersión de tipo F. Un polinomio de grado 10 es el que generó el mínimo error cuadrático. El análisis estadístico de los datos obtenidos, tanto en las simulaciones como en las mediciones experimentales, arrojó una desviación estándar de 8,6 y un error de $0,6 \%$.

La sensibilidad del metamaterial para detectar el índice de refracción de un material depositado dentro de las celdas de la estructura EBG, se calcula como el cociente entre la variación de los parámetros de la brecha medidos (Tabla 2), y la variación del índice de refracción. En la Tabla 3 se presenta la sensibilidad para cada uno de los tres parámetros de la brecha. El parámetro que presentó mayor sensibilidad fue el nivel de pérdidas por inserción.

Para validar la respuesta del prototipo, se seleccionaron dos materiales con índices de refracción teóricos conocidos: el etanol, $n=1.360$, tres lentes con radio de curvatura de 25,44 $\mathrm{mm}^{2}$ y $n=1,5$, y uno con un índice de refracción desconocido, correspondiente a trozos de sustrato RT/ duroid®-6010.

En la Figura 5a se muestra la determinación gráfica del índice de refracción de tres materiales midiendo la variación en $f_{c}$ de la brecha generada. Mediante la herramienta de línea

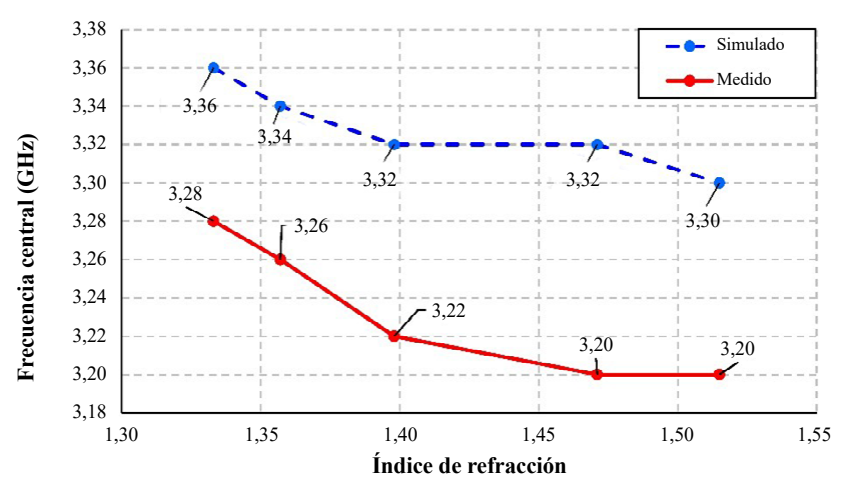

(a)

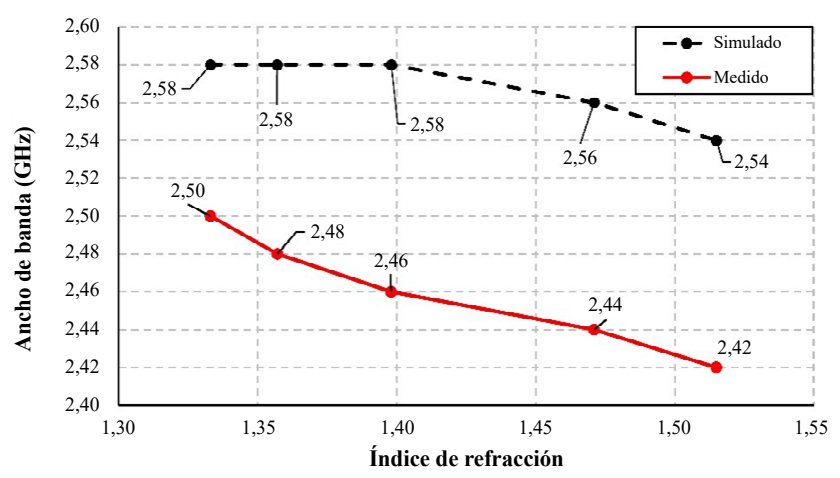

(b)

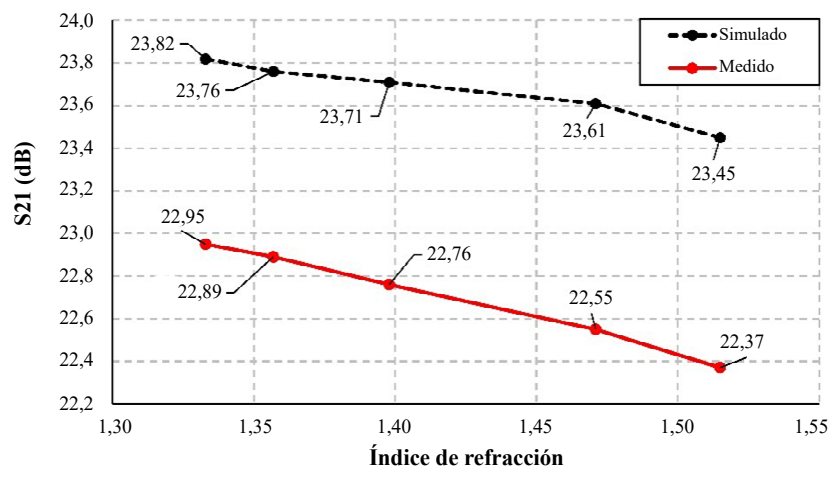

(c)

Figura 3. Variación de la $f_{c}$, el $B W$ y el $I L\left(S_{21}\right)$ de la brecha generada al depositar líquidos con diferentes índices de refracción dentro de la estructura EBG. 
de tendencia de Microsoft Excel, se determinó la ecuación correspondiente a la curva de variación de la frecuencia, lo que dio como resultado un polinomio de grado 3 , y luego se calcularon los datos correspondientes a la variación del índice de refracción en un rango de 1.3 a 1.9. Se ubicaron los datos de la frecuencia central para los tres materiales seleccionados, mostrando las coordenadas de cada punto, donde $x$ es el índice de refracción y $y$ es la $f_{c}$ de la brecha. La medición experimental de la $f_{c}$ se hizo comparando estos datos con los obtenidos mediante simulación.

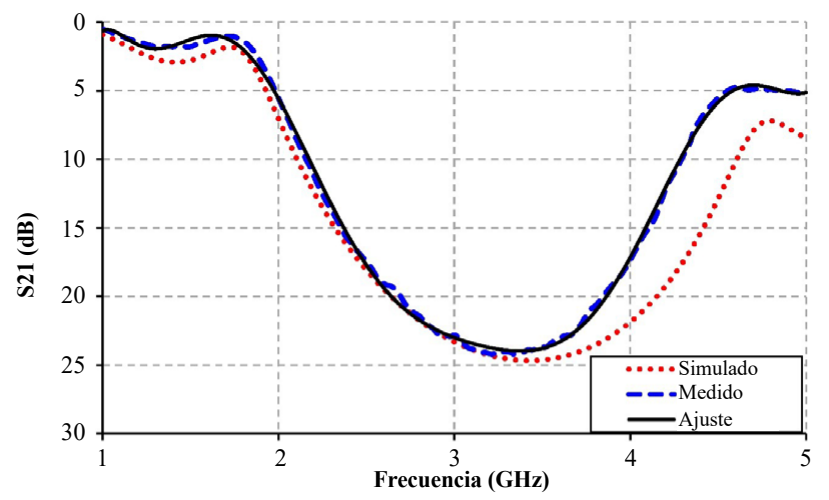

(a)

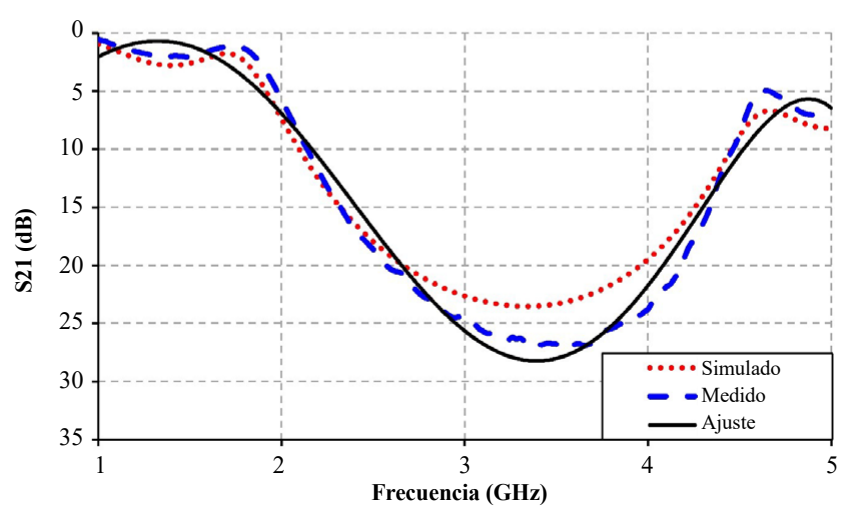

(b)

Figura 4. Parámetro de dispersión $V s$. frecuencia (dato ajustado). (a) Agua destilada y (b) aceite de inmersión de tipo F, dentro de la estructura EBG.

Tabla 3. Sensibilidad de los parámetros de la brecha generada

\begin{tabular}{lccc}
\hline $\begin{array}{l}\text { Parámetro } \\
\text { de la brecha } \\
\text { (bandgap) }\end{array}$ & $\begin{array}{c}\text { Variación del } \\
\text { parámetro }\end{array}$ & $\begin{array}{c}\text { Variación } \\
\text { del índice de } \\
\text { refracción }\end{array}$ & Sensibilidad \\
\hline $\begin{array}{l}\text { Frecuencia } \\
\text { central }\end{array}$ & $0,02 \mathrm{GHZ}$ & 0,182 & 0,11 \\
\hline $\begin{array}{l}\text { Nivel de } \\
\text { pérdidas por } \\
\text { inserción }\end{array}$ & $0,58 \mathrm{~dB}$ & 0,182 & 3,19 \\
$\begin{array}{l}\text { Ancho de } \\
\text { banda }\end{array}$ & $0,08 \mathrm{GHz}$ & 0,182 & 0,44 \\
\hline
\end{tabular}

Con un procedimiento similar se utilizaron los datos del nivel de pérdidas por inserción para calcular el índice de refracción de materiales. El resultado se presenta en la Tabla 4 y en la Figura $5 b$.

\section{Discusión}

Los resultados obtenidos en las simulaciones electromagnéticas y en el procedimiento experimental evidenciaron que la frecuencia central y el nivel de pérdidas por inserción de la brecha generada son los parámetros con la respuesta más lineal ante la variación del índice de refracción del medio por el cual se trasmite la señal de microondas, esto como consecuencia de depositar un material, líquidos en este caso, dentro de las celdas de la estructura EBG. El nivel de pérdidas por inserción es el parámetro que presentó el mayor factor de sensibilidad.

En consecuencia, mediante el monitoreo de la $f_{c}$ y el nivel de pérdidas por inserción, fue posible estimar el índice de refracción de materiales utilizando un metamaterial al depositarlos dentro de las celdas de una estructura EBG periódica.

Sin embargo, las curvas correspondientes al parámetro de dispersión $S_{21}$ logradas, no presentaron un pico definido en el valor de la $f_{c}$ de la brecha, lo que dificultó su estimación

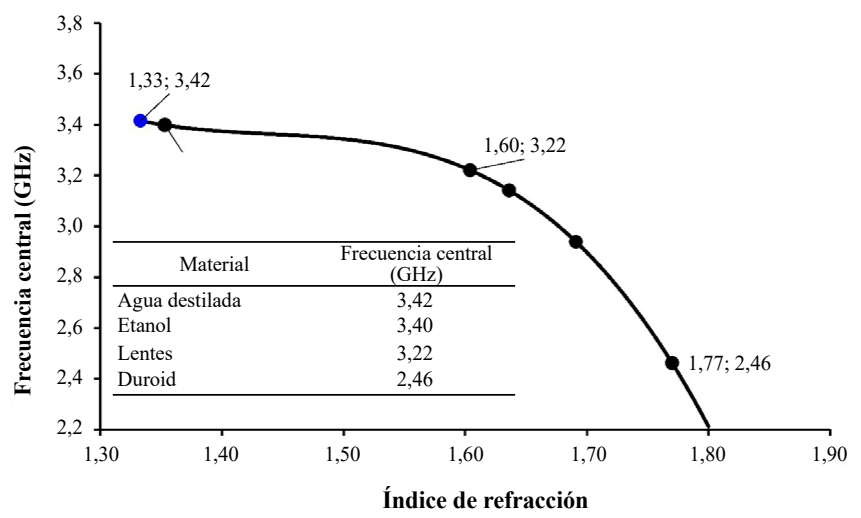

(a)

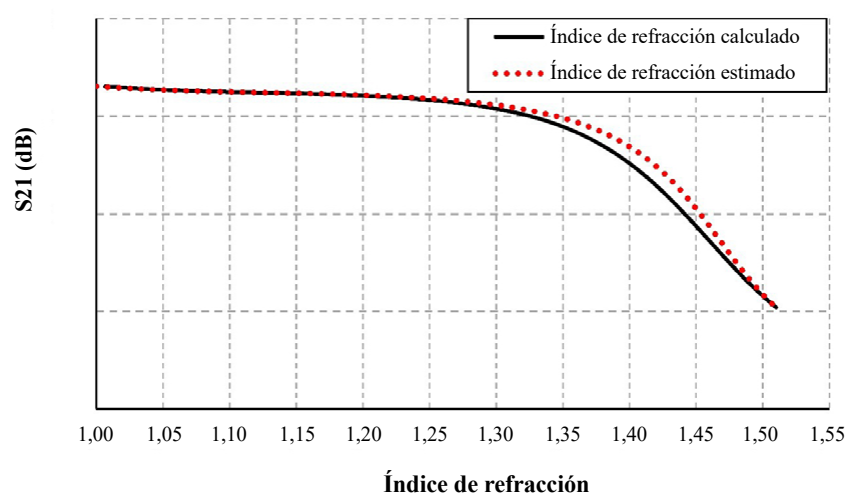

(b)

Figura 5. Estimación del índice de refracción de tres materiales utilizando el metamaterial desarrollado, (a) por variación de la $f_{c}$ y (b) por variación de $S_{21}$. 
Table 4. Estimación del índice de refracción de tres materiales midiendo el parámetro S21

\begin{tabular}{lcccccc}
\hline Material & \multirow{2}{*}{$\begin{array}{c}\text { Índice de } \\
\text { refracción }\end{array}$} & \multirow{2}{*}{$\begin{array}{c}\text { Índice de } \\
\text { refracción estimado }\end{array}$} & \multicolumn{2}{c}{ Frecuencia (GHz) } & \multicolumn{2}{c}{ Pérdidas por Inserción - IL(dB) } \\
\cline { 4 - 7 } & 1,000 & 1,007 & Simulado & Medido & Simulado & Medido \\
\hline Aire & 1,360 & 1,340 & 3,38 & 3,54 & 24,13 & 23,61 \\
Etanol & 1,510 & 1,503 & 3,34 & 3,24 & 22,86 & 22,88 \\
Lentes & & & 3,20 & 23,45 & 22,35 \\
\hline
\end{tabular}

mediante la simple observación. Probablemente, el uso de estructuras típicas de metamateriales, como los anillos resonantes (split-ring resonator, SRR), dentro de las celdas permitan obtener curvas con picos (shift) más definidos y de mayor precisión y facilidad para estimar el valor del parámetro $S_{21}$. El metamaterial desarrollado es de fácil fabricación, no demanda mayores recursos y presenta un buen desempeño en el rango de las microondas.

\section{Material suplementario}

Figure 1S. Prototipo del metamaterial fabricado sobre el sustrato FR4. Vea la figura $1 \mathrm{~S}$ en: https://www.raccefyn.co/index.php/raccefyn/ article/downloadSuppFile/850/3839

Figure 2S. Montaje experimental para medir los parámetros de dispersión del metamaterial usando el analizador VNA. Vea la figura $2 \mathrm{~S}$ en: https://www.raccefyn.co/index.php/raccefyn/article/ downloadSuppFile/850/3840

\section{Contribución de los autores}

Los autores participaron conjuntamente en el desarrollo, simulación y medición de los modelos y prototipos; en la validación y análisis de los resultados, y en la elaboración del manuscrito

\section{Conflicto de interereses}

Los autores manifiestan que no existe ningún tipo de conflicto de intereses en el desarrollo de la investigación, ni en la publicación de los resultados presentados en el presente artículo.

\section{Referencias}

Bakir, M., Karaaslan, M., Unal E., Akgol, O., Sabah, C. (2017). Microwave metamaterial absorber for sensing applications, Opto-Electronics Review. 25: 318-325.

Castro, J.R. \& Ávila, A.G. (2012). Efecto de las características geométricas de estructuras PBG y sus posibles aplicaciones. Tésis de maestría, Universidad de los Andes. Departamento de Ingeniería Eléctrica y Electrónica, Bogotá D.C.
Chen, T., Li, S., Sun, H. (2012). Metamaterials application in sensing. Sensors, 12: 2742-2765. Doi: 10.3390/s120302742

Ciminelli, C. \& Andreone, A. (2011). Introduction to photonic crystals and metamaterials. En Andreone, A., Cusano, A., Cutolo, A., Galdo, V. Selected Topics in Photonic and Metamaterial, p. 1-46. Singapore: Mainland Press Pte Ltd.

Domínguez, M., Cataño, D., Reyes, E. (2015). Design a sensor of relative permittivity of a medium using an antenna microstrip with metamaterials structures. Actas de Ingeniería. 1: 110-114.

Fei, F., Sai, C, Xiang-Hui, W. Peng-Fei, W., Shen-Jiang, C. (2015). Teraherzt refractive index sensor based on photonic column array. IEEE Photonics Technology Letters. 27 (5): 478-481.

Lapine, M., Powell, D., Gorkunov, M., Shadrivov, I., Marqués, R., Kivshar, Y. (2009). Structural tunability in metamaterials. Applied Physics Letters. 95 (8): 1-3.

Liu, W, Fan, F, Chang, S. Hou, J., Chen, M., Wang, Bai, J. (2017). Nanoparticles doped film sensing based on terahertz metamaterials. Optics Communications. 405: 17-21.

Majidifar, S. \& Karimi, G. (2016). New approach for dielectric constant detection using a microstrip sensor. Measurement. 93: $310-314$.

RoyChoudhury, S., Rawat, V., Jalal, A.H., Kale, S.N. (2016). Recent advances in metamaterial split-ring-resonator circuits as biosensors and therapeutic agents. Biosensor and Biolectronics. 86: 595-608.

Sadat, F. \& Ahmadi-S, J. (2017). Industrial liquid characterization enhancement using microwave sensor equipped with electronic band gap structure. International Journal of Electronics and Communications 9 (AEÜ). 82: 152-159.

Shengyong, L., Xiaochuan, A., Ronghua, W., Jiajun, C. (2018) Experimental demonstration of metal-dielectric metamaterial refractive index sensor. Optics and Laser Technology. 100: 304-308.

Tümkaya, M.A., Karaaslan, M., Sabah, C. (2018) Metamaterialbased fluid sensor for identifying different types of fuel oil samples. Chinese Journal of Physics. 56: 1872-1878. Doi: 10.1016/j.cjph.2018.08.018 